\title{
Pseudoxanthoma Elasticum
}

\author{
Wg Cdr S Arora*, Surg Cmde BS Rathore ${ }^{+}$, Lt Col RK Chawla\#, Lt Col P Ranjan**
}

MJAFI 2008; 64 : 70-71

Key Words : Pseudoxanthoma elasticum

\section{Introduction}

$\mathrm{P}$ seudoxanthoma elasticum (PXE) is an inherited multisystem connective tissue disorder in which the elastic fibres present in the skin, eyes, and cardiovascular system gradually become calcified and inelastic. PXE is also known as Gronblad-Strandberg-Touraine syndrome and systemic elastorrhexis. The incidence of PXE is 0.6 per 100,000 people [1].The present case reports highlight the sporadic nature of this disorder, the variable age of presentation and the possibility of an early diagnosis.

\section{Case1}

A 37 year old lady presented with progressive laxity of the skin around the neck of two years duration and yellowish discoloration of the affected areas for the past one year. The patient first noticed several rough patches on the side of her neck which progressively spread to involve the posterior and anterior aspects of the neck. The skin lesions were otherwise asymptomatic. There was no family history of similar complaints and an examination of her two sons revealed normal offspring. On examination there were yellow xanthomatous plaques along the sides and anterior aspects of the neck with lack of elasticity. Ophthalmological and cardiovascular examinations were normal. Skin biopsy done from the side of the neck revealed fragmented elastic fibres in the reticular dermis.

Case 2

A six year old girl was brought by her parents with yellowish papular lesions over anterior aspect of the neck and larger yellow lesions in both axillae for the past one year. The skin lesions were otherwise asymptomatic. There was no family history of similar illness. Examination revealed a chicken skin appearance of the neck with yellow papules and plaques in both the axillae and neck (Figs. 1,2). There was no cutaneous laxity. Eye and cardiovascular examination were normal. Skin biopsy done from the axillary plaque revealed elastin fibre degeneration in the reticular dermis (Fig. 3).

\section{Discussion}

Pseudoxanthoma elasticum is a genetically inherited disorder of elastic tissue characterized by abnormal calcification and progressive degeneration of elastic tissue in multiple organs, including skin, gastrointestinal tract, eyes, and the cardiovascular system. Skin manifestations are quite distinctive. Small waxy yellow papules typically appear in the skin in early adolescence, giving the appearance of "plucked chicken skin". The lesions have a predilection for flexural areas and the neck and axillae but over a period of time these become generalized. With time, the skin becomes lax and hangs in folds and clinically resembles cutis laxa. Our first case presented with this typical history and progression of disease at the age of 37 years. The second case was diagnosed at an early stage at the age of six years.

The vascular calcification and altered elastin within the gastrointestinal (GI) tract results in haemorrhage early in life, commonly from the upper GI tract. Angioid streaks, which occur in Bruch's membrane, are seen in the eyes of most patients, as are retinal haemorrhage and detachment. Vascular alterations result in peripheral vascular disease, cerebral accidents, premature myocardial infarction and hypertension subsequent to renal artery involvement.

Pseudoxanthoma elasticum is divided into Type I and Type II. Inheritance may be dominant or recessive. The classic Type I recessive form has the highest incidence of GI involvement, whereas Type II has minimal or no visceral involvement [2]. Genetic consultation and counselling are important in determining prognosis and potential complications later in life. Although the pathogenesis is not fully understood, there seems to be progressive calcification, fragmentation and clumping of elastin fibres. The degradation of this abnormal elastin results in deposition of proteoglycans in the skin and

"Graded Specialist (Derm \& Ven), 5 Air Force Hospital, C/o 99 APO. +PDMS, O/o DGMS Navy, NHQ, New Delhi. "Classified Specialist (Derm \& Ven),Military Hospital, Bareilly. ${ }^{* *}$ Classified Specialist (Pathology), Base Hospital Delhi Cantt. 


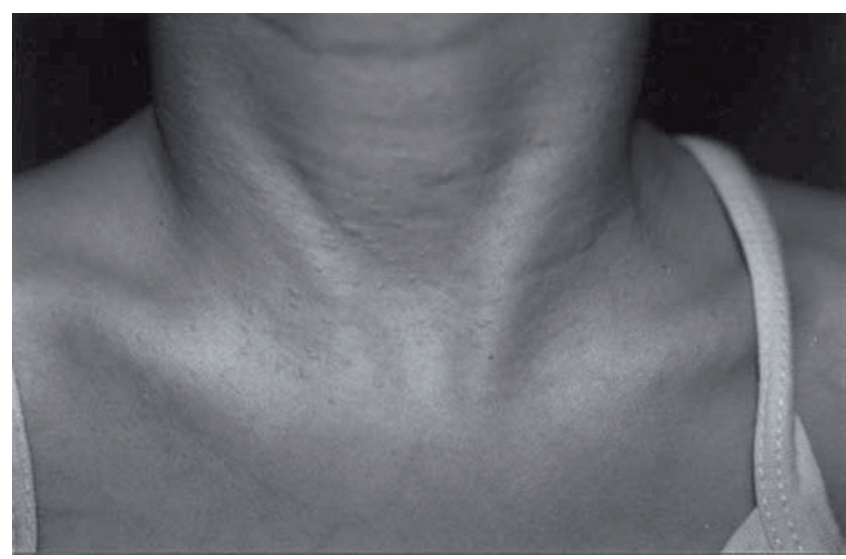

Fig. 1 : Yellow papules on anterior aspect neck

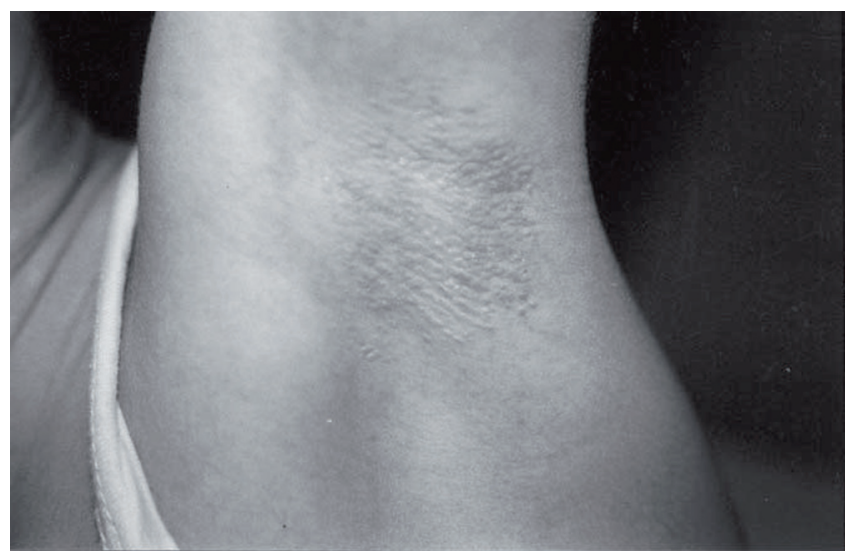

Fig. 2: Yellow papules and plaques left axillary region

urine of patients [3]. In both of our cases there was no positive family history of similar complaints.

Hereditary disorders often have a prolonged time lapse between clinical onset and diagnosis. However, the delay in diagnosis of PXE is unusually long, averaging about nine years [4] which in our case was less than two years. The late diagnosis makes early intervention difficult and has important implications for genetic counselling. The disease is often not diagnosed until the fourth or fifth decade of life because the clinical manifestations and complications have not become noticeable or are not severe enough to warrant medical attention. In one study, most patients who sought medical opinion for PXE skin lesions from different non dermatologist physicians were told that the skin disorder did not appear to be harmful [4]. The earliest reported case was of an infant who reportedly had recognizable skin lesions at birth

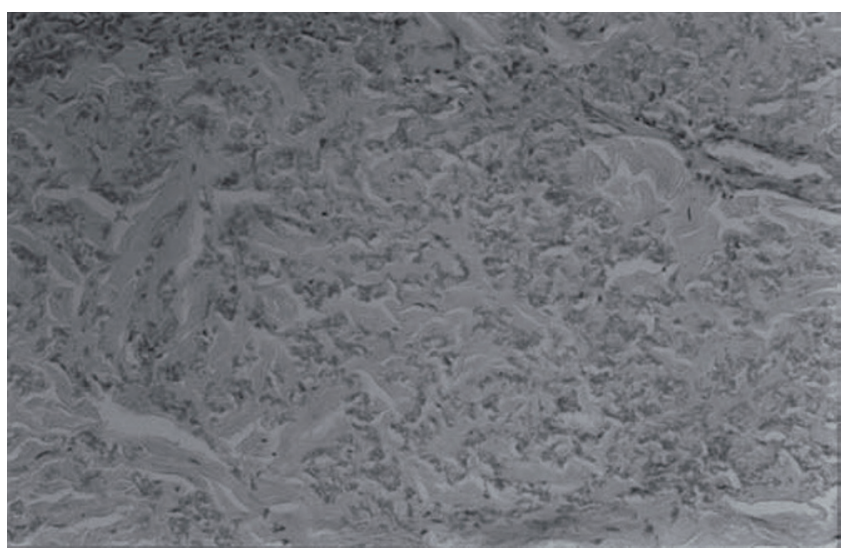

Fig. 3: High power photomicrograph showing elastin fibre degeneration

according to the parents, although there was no documented physical examination or skin biopsy to confirm this [5] and the oldest patient reported has been 75 years old [6] at the time of diagnosis.

The first patient showed a progression of skin lesions during the first six monthly follow up. A re-examination did not reveal any systemic involvement. She was thereafter lost to follow up. Our second case has adhered to the follow up protocol. At the end of two years follow up she has a mild increase in her skin lesions with absence of any systemic involvement.

\section{Conflicts of Interest}

None identified

\section{References}

1. Ward SK, Roenigk H, Gordon KB. Dermatologic manifestations of gastrointestinal disorders. Gastroenterol Clin North Am 1998; 27:615-36.

2. Gregory B, Ho V. Cutaneous manifestations of gastrointestinal disorders Part II. J Am Acad Dermatol 1992;26:371-83.

3. Wenstrup RJ, Pinnell SR. Heritable disorders of connective tissue with skin changes. In: Fitzpatrick TB, Eisen AZ, Wolff K, Freedberg IM, Austen KF, editors. Dermatology in General Medicine. New York: McGraw-Hill, 1993; 1961-74.

4. Neldner KH. Pseudoxanthoma elasticum. Clin Dermatol 1988;6:1-159.

5. Goodman RM, Smith EW, Paton D, Bergman RA, Siegel CL, Ottesen OE, et al. Pseudoxanthoma elasticum: A clinical and histopathological study. Medicine 1963; 42:297-334.

6. Sherer DW, Bercovitch L, Lebwohl M. Pseudoxanthoma elasticum: Significance of limited phenotypic expression in parents of affected offspring. J Am Acad Dermatol 2001; 44 : 534-7. 